\title{
IncRNA SNHG5 is associated with poor prognosis of bladder cancer and promotes bladder cancer cell proliferation through targeting $p 27$
}

\author{
ZHIPENG MA $^{1}$, SENYAO XUE ${ }^{1}$, BI ZENG ${ }^{2}$ and DAOXIAN QIU ${ }^{1}$ \\ ${ }^{1}$ Department of Urology, Yidu Central Hospital of Weifang; ${ }^{2}$ Department of Obstetrics and Gynecology, \\ The People's Hospital of Qingzhou, Weifang, Shandong 262500, P.R. China
}

Received April 6, 2017; Accepted November 20, 2017

DOI: $10.3892 / \mathrm{ol} .2017 .7527$

\begin{abstract}
Long non-coding RNAs (lncRNAs) have been identified as critical regulators in tumorigenesis. In our present study, we measured the level of small nucleolar RNA host gene 5 (SNHG5) in bladder cancer (BC) tissues and cell lines, and the correlation of the level of SNHG5 with clinicopathological features and prognosis of BC patients was analyzed. Reverse transcription-quantitative polymerase chain reaction was performed to determine the level of SNHG5 in the BC tissues and cell lines. The Kaplan-Meier method was used to analyze the long-term survival outcomes. MTT and colony formation assays were applied to assess the influence of SNHG5 on cell proliferation ability. Flow cytometry was used to measure the function of SNHG5 on cell cycle and apoptosis rate. SNGH5 was found upregulated in BC tissues and cell lines and a high level of SNGH5 was correlated with a poor prognosis. Silencing $S N H G 5$ inhibited the proliferation ability of BC cells and such a function was attributed to its influence on cells cycle and apoptosis. Our findings imply that SNHG5 was upregulated in $\mathrm{BC}$ tissues and played an important role in $\mathrm{BC}$ progression and may be a potential therapeutic target for BC patients.
\end{abstract}

\section{Introduction}

Bladder cancer (BC), one of the most prevalent carcinomas worldwide, has been identified as the fourth and tenth leading cause of cancer-related deaths in males and females, respectively (1). Despite many efforts have been made, the prognosis still remains unsatisfied. The initiation and progression of $\mathrm{BC}$ involves changes about a variety of oncogenes and tumor

Correspondence to: Dr Daoxian Qiu, Department of Urology, Yidu Central Hospital of Weifang, 4138 South Linglongshan Road, Qingzhou, Weifang, Shandong 262500, P.R. China

E-mail: qiudaoxian0921@163.com

Key words: bladder cancer, small nucleolar RNA host gene 5, proliferation, cell cycle, apoptosis suppressors. Therefore, investigating the molecular mechanisms underlying the tumorigenesis of bladder cancer cells is essential for exploring novel treatment targets.

Currently, long non-coding RNAs (lncRNAs), newly identified members of the noncoding RNA family with length $>200$ nucleotides (nt), have been proposed (2-4). Accumulating documents have revealed that lncRNAs play a critical role in tumorigenesis and can be used as biomarkers for diagnosis or prediction of survival and recurrence in multiple cancers (5-9). For instance, in 2017, Idogawa et al reported that long non-coding RNA NEAT1 was a transcriptional target of $p 53$ and modulated $p 53$-induced transactivation and tumor-suppressor function (10). Zhou et al demonstrated that downregulation of lncRNA MEG3 mediated by DNMT3b contributed to nickel malignant transformation of human bronchial epithelial cells via modulating PHLPPI transcription and HIF-1 $\alpha$ translation (11). Wang et al uncovered that 2-O-Methylmagnolol upregulated the long non-coding RNA, GAS5, and enhanced apoptosis in skin cancer cells (12). Several other studies also demonstrated the function of lncRNAs in BC (13-15). Despite so many lncRNAs have been reported to be associated with $\mathrm{BC}$, still many lncRNAs need to be investigated.

Small nucleolar RNA host gene 5 (SNHG5), a SnoRNA-U50-associated lncRNA, has been demonstrated downregulated in bladder cancer (BC) and colorectal carcinoma (CRC) $(16,17)$. However, its biological function in $\mathrm{BC}$ has not been investigated. The aim of our present study is to investigate whether SNGH5 is associated with BC progression and to identify the role of SNHG5 in the prognosis of BC. Herein, we uncovered that SNHG5 was significantly overexpressed in BC tissues which was associated with larger tumor range, metastasis, lymph nodes, pathological stage and poor prognosis. In addition, we demonstrated that silenced SNHG5 suppressed cell proliferation through influencing cell cycle and apoptosis rate. Therefore, the results indicated that SNHG5 acted as an oncogene in BC.

\section{Materials and methods}

Patients and clinical samples collection. BC tissues $(n=67)$ and pair-matched noncancerous tissues were obtained through tissue biopsy from patients diagnosed with $\mathrm{BC}$ at the 
Department of Urology, Yidu Central Hospital of Weifang between March 2013 and September 2016. Informed consent was obtained from patients. All procedures involving human participants were in accordance with the ethical standards of the Human Research Ethics Committee at the Department of Urology, Yidu Central Hospital of Weifang.

Cell lines. Bladder cancer SW780, UMUC3, 5637, T-24 and one normal urothelial cell line SVHUC-1 utilized in present study were purchased from the Tumor Cell Bank of the Chinese Academy of Medical Science (Shanghai, China). The UMUC3, T24 and SV-HUC-1 cells were cultured in Dulbecco's modified Eagle's medium (DMEM; Invitrogen Life Technologies, Carlsbad, CA, USA) plus $10 \%$ fetal bovine serum and ampicillin and streptomycin at $37^{\circ} \mathrm{C}$ in a humidified atmospherewith 95\% air and 5\% $\mathrm{CO}_{2}$. The 5637 and SW780 cells were cultured in RPMI-1640 medium (Invitrogen Life Technologies) plus $10 \%$ fetal bovine serum and ampicillin and streptomycin at $37^{\circ} \mathrm{C}$ in a humidified atmosphere with $95 \%$ air and $5 \% \mathrm{CO}_{2}$.

Reverse-transcription quantitative polymerase chain reaction ( $R T-q P C R)$. Total RNAs from tissues and cells were isolated with TRIzol reagent (Invitrogen Life Technologies) under the manufacturer's instructions. Reverse transcription was performed with PrimeScript RT reagent kit (Takara Bio, Inc., Otsu, Japan) according to the manufacturer's instructions. RT-qPCR was performed with SYBR Prime Script RT-PCR kits (Takara Bio, Inc.) based on the manufacturer's instructions. The $S N H G 5$ level was calculated with the $2^{-\Delta \Delta C t}$ method, which was normalized to GAPDH mRNA. The primers for SNHG5 were as the following: forward, 5'-CGCTTGGTT AAAACCTGACACT-3' and reverse, 5'-CCAAGACAATCT GGCCTCTATC-3'; the primers for $G A P D H$ were as the listed: Forward, 5-ACGGGAAGCTCACTGGCATGG-3' and reverse, 5'-GGTCCACCACCCTGTTGCTGTA-3'. All assays were performed in triplicate. The expression levels were relative to the fold change of the corresponding controls, which were defined as 1.0.

siRNA transfections. Cells were transfected with siRNAs for SNHG5 by using Lipofectamine 2000 (Invitrogen Life Technologies) according to the manufacturer's instructions. After transfection (48 h), cells were harvested for the following experiments including RT-qPCR, western blot analysis, proliferation assays and flow cytometry. RNA oligonucleotides were purchased from GenePharma (Shanghai, China). The siRNA sequence for SNHG5 was si-SNHG5, 5'-CCTCTGGTCTCA TCTGCATATTGACTTA-3'.

Cell viability. Cell viability was assessed via 3-(4,5-dimethylthiazol-2-yl)-2,5-diphenyl-trtrazolium bromide (MTT) assay. $5 \times 10^{3}$ cells/well transfected with indicated vector were seeded in a 96-well flat-bottomed plate for $24 \mathrm{~h}$ and cultured in a normal medium. At 0, 24, 48, 72 and $96 \mathrm{~h}$ after transfection, the MTT solution $(5 \mathrm{mg} / \mathrm{ml}, 20 \mu \mathrm{l})$ was added to each well. Following incubation for $4 \mathrm{~h}$, the media was removed and $100 \mu \mathrm{l}$ of DMSO was added to each well. The relative number of surviving cells was assessed by measuring the optical density (OD) of cell lysates at $560 \mathrm{~nm}$. All assays were performed in triplicate.
Table I. Correlation between SNHG5 expression and clinical features $(n=67)$.

\begin{tabular}{|c|c|c|c|}
\hline \multirow[b]{2}{*}{ Variable } & \multicolumn{2}{|c|}{$\begin{array}{c}\text { SNHG5 } \\
\text { expression }\end{array}$} & \multirow[b]{2}{*}{ P-value } \\
\hline & Low & High & \\
\hline Age (years) & & & 0.142 \\
\hline$<60$ & 9 & 17 & \\
\hline$\geq 60$ & 22 & 19 & \\
\hline Sex & & & 0.088 \\
\hline Male & 19 & 14 & \\
\hline Female & 12 & 22 & \\
\hline Smoking & & & 0.820 \\
\hline No smoking & 8 & 17 & \\
\hline Smoking & 23 & 19 & \\
\hline Tumor range & & & 0.001 \\
\hline T1-T3 & 22 & 10 & \\
\hline$\geq \mathrm{T} 4$ & 9 & 26 & \\
\hline Metastasis & & & 0.013 \\
\hline Negative & 23 & 15 & \\
\hline Positive & 8 & 21 & \\
\hline Lymph nodes & & & 0.001 \\
\hline Negative & 20 & 9 & \\
\hline Positive & 11 & 27 & \\
\hline Pathological stage & & & 0.003 \\
\hline$<\mathrm{IV}$ & 21 & 11 & \\
\hline$\geq \mathrm{IV}$ & 10 & 25 & \\
\hline
\end{tabular}

Low/high by the sample median. Pearson $\chi^{2}$ test. $\mathrm{P}<0.05$ was considered statistically significant. SNHG5, small nucleolar RNA host gene 5.

Colony formation assay. Cells (500 cells/well) transfected with indicated vector were plated in 6-well plates and incubated in RPMI-1640 with $10 \% \mathrm{FBS}$ at $37^{\circ} \mathrm{C}$. Two weeks later, the cells were fixed and stained with $0.1 \%$ crystal violet. The number of visible colonies was counted manually.

Flow cytometric analysis of apoptosis and cell cycle distribution. Apoptosis was performed by using flow cytometric analysis with Annexin V: FITC Apoptosis Detection kits (BD Biosciences, San Diego, CA, USA), according to the manufacturer's instructions. For cell cycle distribution, cells were collected directly or $48 \mathrm{~h}$ after transfection and washed with ice-cold phosphate-buffered saline (PBS), and fixed with $70 \%$ ethanol overnight at $-20^{\circ} \mathrm{C}$. Fixed cells were rehydrated in PBS for $10 \mathrm{~min}$ and incubated in RNase A $(1 \mathrm{mg} / \mathrm{ml})$ for $30 \mathrm{~min}$ at $37^{\circ} \mathrm{C}$, then the cells were subjected to PI/RNase staining followed by flow cytometric analysis with a FACScan instrument and CellQuest software (both from Becton-Dickinson, Mountain View, CA, USA) as described (20).

Western bolt analysis and antibodies. Total protein lysates were separated in $10 \%$ sodium dodecyl sulfate-polyacrylamide gel electrophoresis (SDS-PAGE), and were electrophoretically transferred to polyvinylidene difluoride 
A

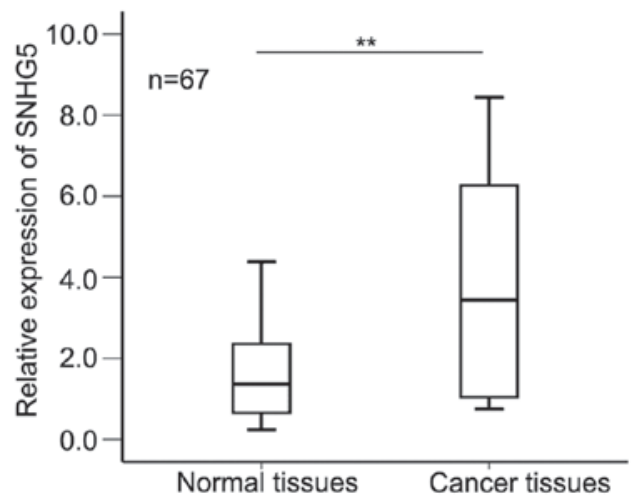

B

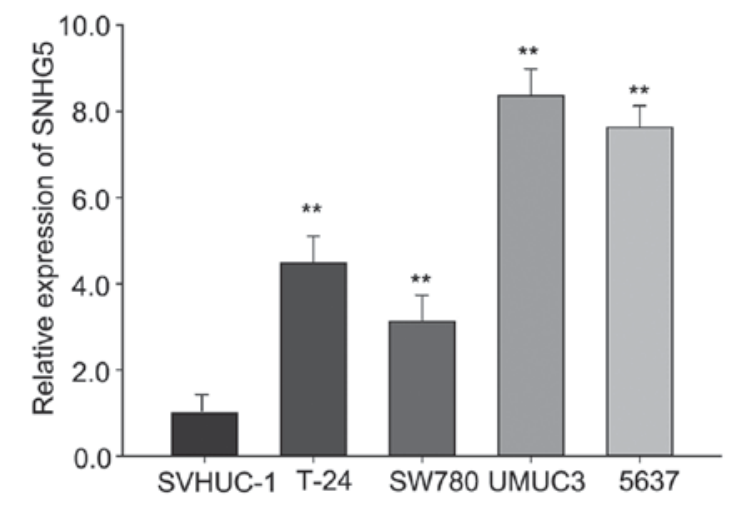

Figure 1. SNHG5 was upregulated in BC tissues and cell lines. (A) RT-qPCR was performed to measure the level of SNHG5 in BC tissues and corresponding normal tissues. (B) The level of SNHG5 in BC cell lines SW780, UMUC3, 5637, T-24 and one normal urothelial cell line SVHUC-1 was detected by RT-qPCR. Error bars represented the mean \pm standard deviation of at least three independent experiments. ${ }^{*} \mathrm{P}<0.05$, ${ }^{* *} \mathrm{P}<0.01$ vs. control group. SNHG5, small nucleolar RNA host gene 5; RT-qPCR, reverse transcription-quantitative polymerase chain reaction; BC, bladder carcinoma.

membranes (Roche Diagnostics, Indianapolis, IN, USA). Protein loading was estimated by using mouse anti-GAPDH monoclonal antibody. The membranes were blotted with $10 \%$ non-fat milk in TBST for $2 \mathrm{~h}$ at room temperature, washed and then probed with the rabbit anti-human $p 27$ (1:2,000 dilution), CDK2 (1:2,000 dilution), activated caspase-3 (1:2,000 dilution), activated caspase-9 (1:2,000 dilution), and GAPDH (1:3,000 dilution), overnight at $4^{\circ} \mathrm{C}$, followed by treatment with secondary antibody conjugated to horseradish peroxidase for $2 \mathrm{~h}$ at room temperature. The proteins were detected by using an enhanced chemiluminescence system and then exposed to x-ray film. All antibodies were purchased from Abcam (Cambridge, MA, USA).

Statistical analysis. Data were shown as the means \pm standard error of at least three independent experiments. The SPSS 17.0 software (SPSS Inc., Chicago, IL, USA) was used for statistical analysis. Two group comparisons were performed with a Student's t-test. Multiple group comparisons were analyzed with one-way ANOVA. The Pearson $\chi^{2}$ test was used to evaluate the relationship between $S N H G 5$ expression and clinical features. Kaplan-Meier method was used to compare the overall survival curves between high-SNHG5 and low-SNHG5 expression groups via the log-rank test. $\mathrm{P}<0.05$ was considered to indicate a statistically significant difference.

\section{Results}

SNHG5 was upregulated in BC tissues and cell lines. To explore the biological function of $S N H G 5$ in BC, we first measured the level of $S N H G 5$ in BC tissues and corresponding normal tissues ( $\mathrm{n}=67$ ) by RT-qPCR. As shown in Fig. 1A, SNHG5 was aberrantly increased $(\mathrm{P}<0.01)$ in tumor tissues compared with that in corresponding normal tissues. Furthermore, the levels of SNHG5 in four bladder cancer cells SW780, UMUC3, 5637, T-24 and one normal urothelial cell line SVHUC-1 were assessed. As presented in Fig. 1B, the level of SNHG5 was significantly increased in four $\mathrm{BC}$ cell lines in comparison to that in the normal urothelial cell line. And among these cell lines, the expression level of SNHG5 in UMUC3 and 5637 cell

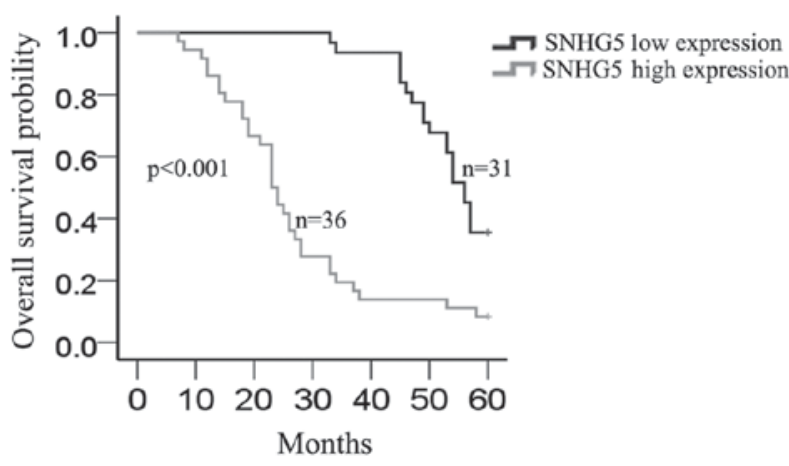

Figure 2. The correlation between SNHG5 and overall survival of BC patients was analyzed by Kaplan-Meier method analysis (log-rank test), $\mathrm{P}=0.000$. SNHG5, small nucleolar RNA host gene 5; BC, bladder carcinoma.

was relative higher than that in SW780 and T-24 cells; therefore, we chose UMUC3 and 5637 as the study object in the following assays. These data revealed that $S N H G 5$ may play a pivotal role in $\mathrm{BC}$ progression.

Correlation of SNHG5 expression with clinicopathological features and prognosis. Then we investigated the relationship between $S N H G 5$ expression and clinicopathological features in BC, the mean expression level of $S N H G 5$ in all BC tissues was used as a cutoff value, and all samples were divided into two groups (high expression group, $\mathrm{n}=36$ vs. low expression group, $\mathrm{n}=31$ ). As illustrated in Table I, high expression level of SNHG5 was significantly correlated with larger tumor range $(\mathrm{P}=0.001)$, metastasis $(\mathrm{P}=0.013)$, lymph nodes $(\mathrm{P}=0.001)$ and pathological stage $(\mathrm{P}=0.003)$, but it had no significant correlation with age, sex and smoking ( $\mathrm{P}>0.05)$. Furthermore, Kaplan-Meier method analysis (log-rank test) was performed to determine the association between SNHG5 expression and overall survival of patients. As shown in Fig. 2, patients with high expression level of SNHG5 had a significantly shorter overall survival than those with low level of $S N H G 5(\mathrm{P}=0.000)$.

Silenced SNHG5 suppresses the proliferation of BC cells. To investigate the biological function of SNHG5 on the 
A
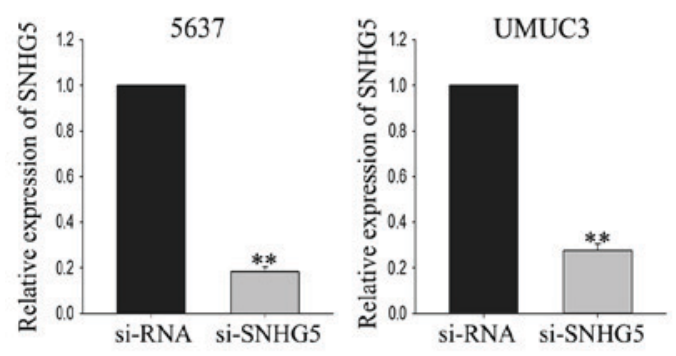

B

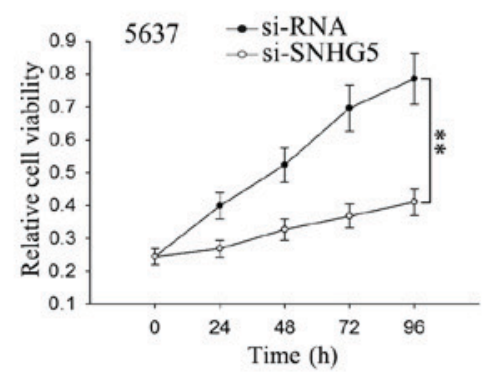

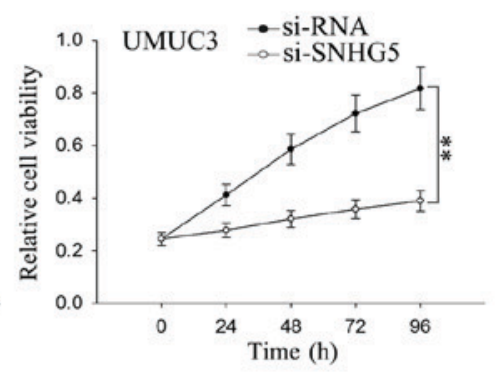

C
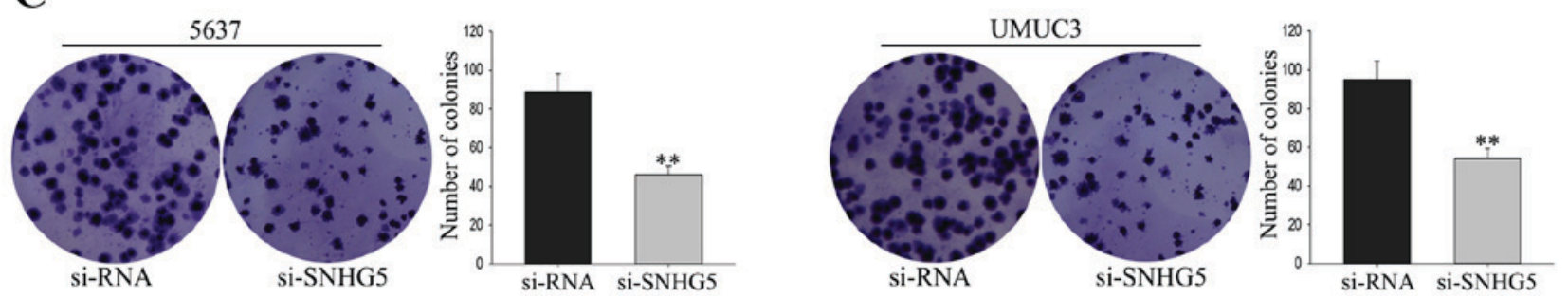

Figure 3. Silenced SNHG5 suppresses BC cell proliferation. (A) The transfection efficiency was obtained from reverse transcription-quantitative polymerase chain reaction after $48 \mathrm{~h}$. (B) The effect of SNHG5 on cell viability was measured by MTT. (C) Colony formation assay was performed to measure the influence of $S N H G 5$ on cell proliferation ability. Error bars represented the mean \pm standard deviation of at least three independent experiments. ${ }^{*} \mathrm{P}<0.05,{ }^{* *} \mathrm{P}<0.01$ vs. control group. SNHG5, small nucleolar RNA host gene 5; BC, bladder carcinoma.

proliferation of BC cells, UMUC3 cell and 5637 cell were transfected with si-SNHG5 andsiRNA was used as as negative control (NC). The satisfactory transfection efficiency was obtained at $48 \mathrm{~h}$ post-transfection (Fig. 3A). MTT assay was performed to measure the function of SNHG5 on cell viability. As shown in Fig. 3B, weakened proliferation ability was obtained from UMUC3 cell and 5637 cell transfected with si-SNHG5 in comparison to the NC-transfected cells. Consistent with the results of MTT, colony formation assay revealed a growth-inhibition effect mediated by si-SNHG5 (Fig. 3C). The findings indicated that silenced SNHG5 could suppress the proliferation of BC cells.

Silenced SNHG5 induces cell cycle arrest at G1 phase and promotes cell apoptosis. To investigate the underlying mechanism of si-SNHG5-mediated growth-inhibition, flow cytometric analysis of cell cycle distribution were performed. As illustrated in Fig. 4A, silenced SNHG5 in UMUC3 cell and 5637 cell obviously induced cell cycle arrest at G1. And flow cytometric analysis of apoptosis revealed that silenced SNHG5 significantly increased the apoptosis rate of UMUC3 cell and 5637 cell (Fig. 4B). Furthermore, western blot assay demonstrated that the level of cyclin-dependent kinase 2 (CDK2) was decreased while the level of $p 27$ was significantly increased; and apoptosis-related proteins (activated caspase- 3 and activated caspase-9) were increased when $S N H G 5$ was knockdown (Fig. 4C). These data indicated that SNHG5 contributed to the proliferation ability of $\mathrm{BC}$ cells, which might be attributed to its influence on cell cycle and apoptosis.

The oncogenic function of SNHG5 is in a p27-dependent manner. To determine whether p27 was involved in the si-SNHG5-mediated growth inhibition, we first measured the level of $p 27$ in BC tissues and corresponding normal tissues by RT-qPCR and then western blot analysis was performed to determine the protein level of $p 27$ in four pairs of cancer tissues and normal tissues. As illustrated in Fig. 5A and B, the mRNA level of p27 was significantly downregulated in BC tissues and the protein level was also obviously decreased in BC tissues. Furthermore, rescue assays were performed to verify whether SNHG5 regulated $\mathrm{BC}$ cell proliferation via silencing $p 27$ expression. UMUC3 and 5637 cells were co-transfected with si-SNHG5 and si-p27, and results from MTT and colony-formation assays revealed that co-transfection with si-p27 could partially abolish the si-SNHG5-mediated growth-inhibition (Fig. 5C). Additionally, flow cytometric analyses of apoptosis and cell cycle distribution showed that co-transfection with si-p27 could partially rescue the si-SNHG5-mediated cell cycle arrest and increased apoptosis rate (Fig. 5D and E). These results indicated that the effect of SNHG5 on BC was partially involved with targeting $p 27$.

\section{Discussion}

Accumulating documents have demonstrated that the dysregulation of lncRNAs are associated with tumorigenesis and progression of malignant tumors (18-23). For instance, Zhou et al demonstrated that downregulation of lncRNA MEG3 mediated by DNMT3b contributed to nickel malignant transformation of human bronchial epithelial cells via modulating PHLPPI transcription and HIF-lalpha translation (11). Zhang et al revealed that long non-coding RNA FTH1P3 facilitated oral squamous cell carcinoma progression by acting as a molecular sponge of $m i R-224-5 p$ to modulate fizzled 5 expression (24). Cui et al uncovered that upregulated lncRNA SNHG1 contributed to progression of non-small cell lung cancer through inhibition of $m i R-101-3 p$ and activation of $W n t / \beta$-catenin signaling pathway (25). SNHG5 is anomalously expressed in 
A

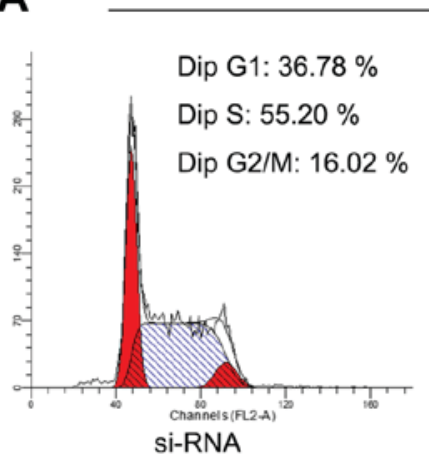

5637

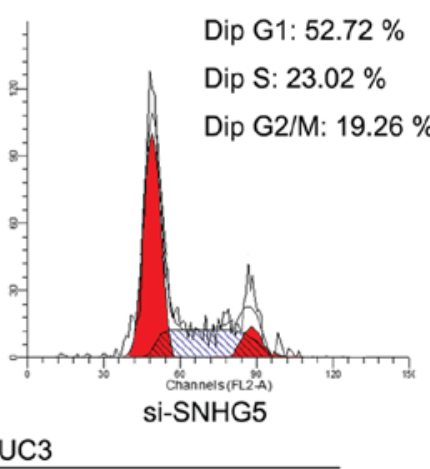

UMUC3
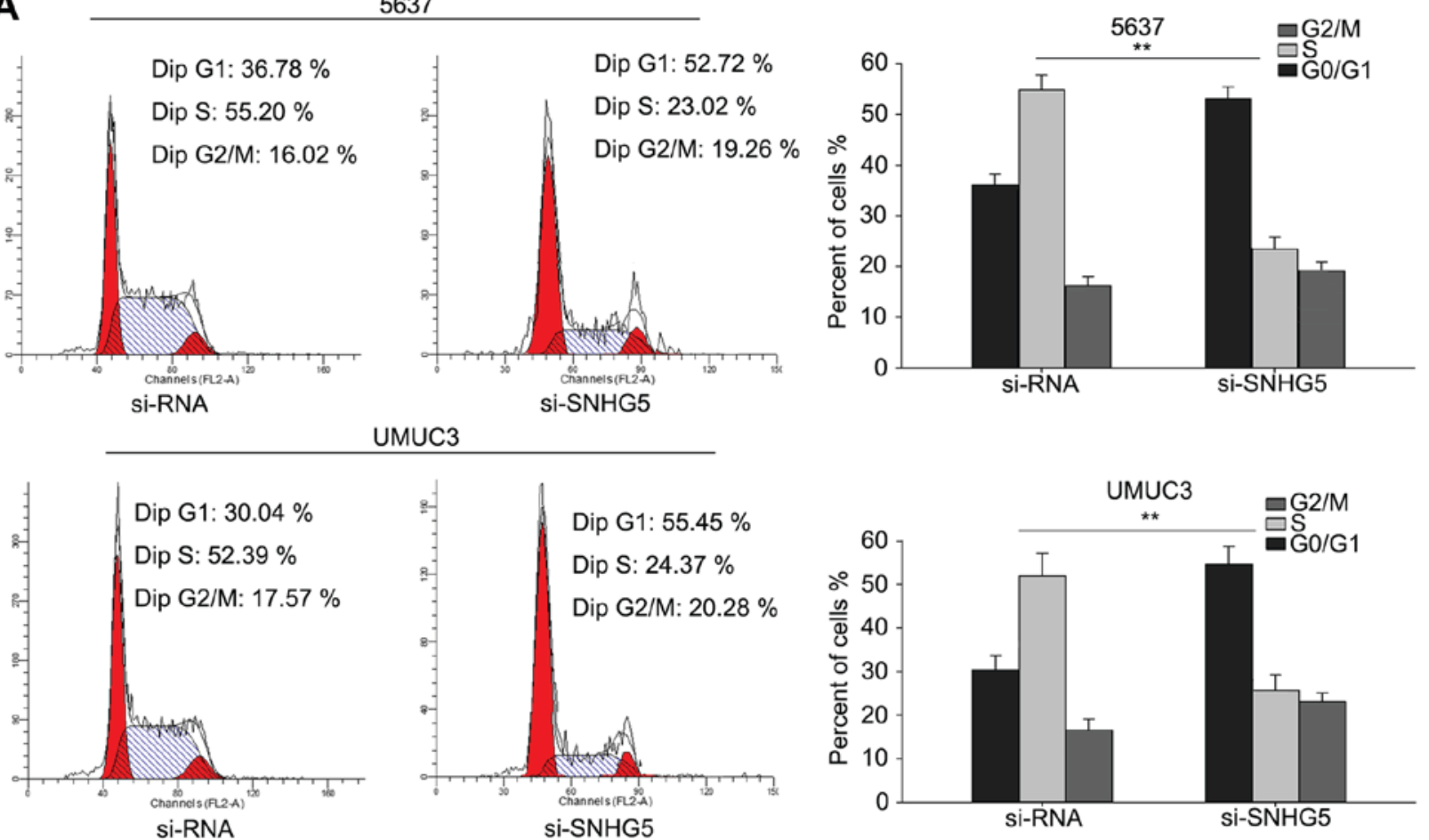

B
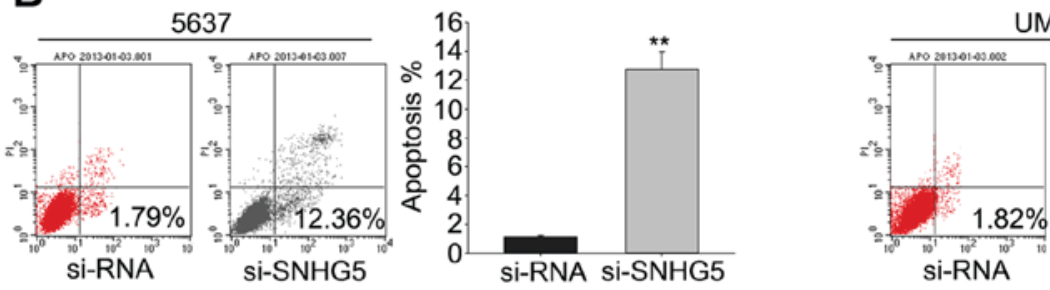

UMUC3
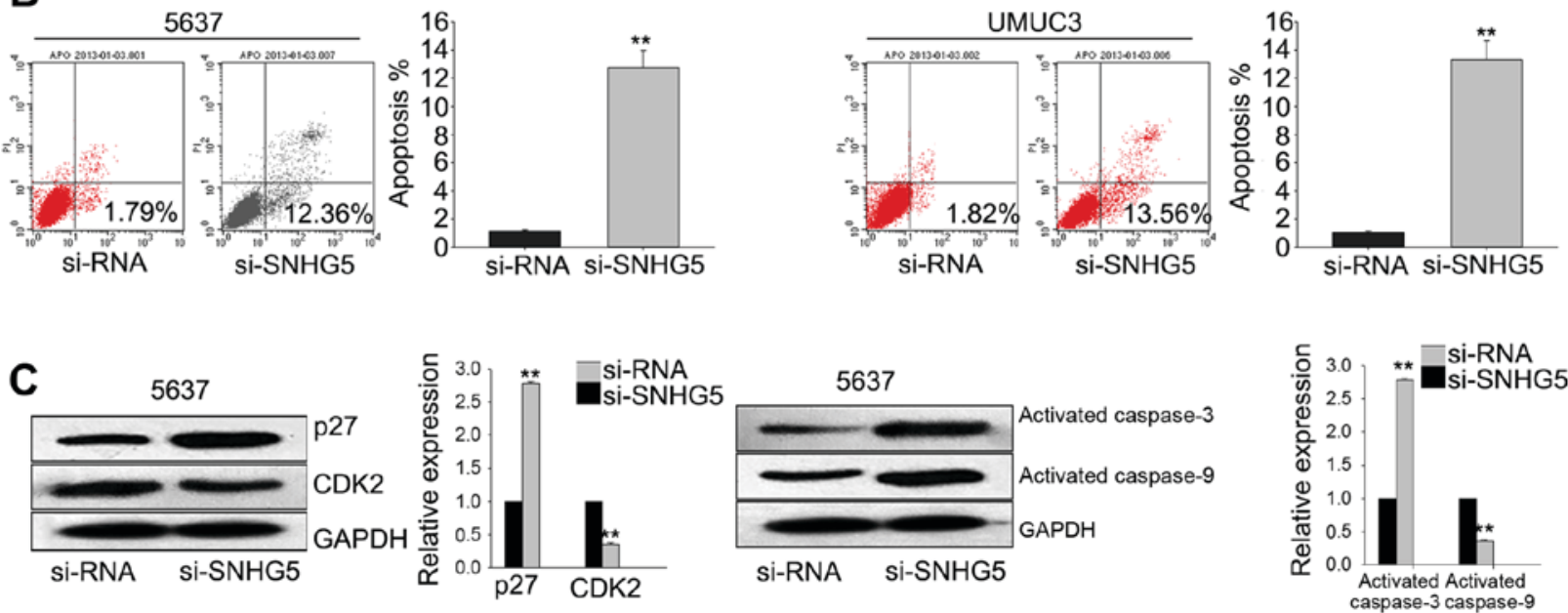

Figure 4. Silenced SNHG5 induces cell cycle arrest at G1 phase and promotes cell apoptosis. Flow cytometric analyses were employed to measure the function of SNHG5 on (A) cell cycle distribution and (B) cell apoptosis rate. (C) Western blot analysis was utilized to detect the expression level of $C D K 2$, p27 and apoptosis-related proteins (activated caspase-3 and activated caspase-9) in the cells after the knockdown of SNHG5. Error bars represented the mean \pm standard deviation of at least three independent experiments. ${ }^{*} \mathrm{P}<0.05,{ }^{* *} \mathrm{P}<0.01$ vs. control group. SNHG5, small nucleolar RNA host gene 5 ; CDK2, cyclin-dependent kinase 2; p27, cyclin-dependent kinase inhibitor 1B; si, small interfering.

human gastric cancer and colorectal cancer $(16,17)$. However, its biological function in $\mathrm{BC}$ has not been investigated.

In our present study, we demonstrated that the level of SNHG5 was significantly increased in BC specimens and $\mathrm{BC}$ cell lines. And analysis of the clinicopathological characteristics of patients with $\mathrm{BC}$ revealed that high level of SNHG5 was associated with tumor range, metastasis, lymph nodes, pathological stage and poor prognosis. These results suggested that SNHG5 expression might be associated with the level of malignancy of $\mathrm{BC}$, and might be involved in the tumorigenesis and progression of $\mathrm{BC}$. It has been demonstrated that the effect of SNHG5 is related with its biological function on cell proliferation $(16,17)$. Therefore, we explored the biological function of SNHG5 in BC cells. We employed MTT and colony formation assays to measure the function of SNHG5 on cell proliferation ability and found that silenced SNHG5 significantly reduced cell growth in BC cells. Then, flow cytometric analysis revealed that the si-SNHG5-mediated growth-inhibition was attributed to its influence on cell cycle and apoptosis rate.

It has been reported that $S N H G 5$ acts as a tumor suppressor in gastric cancer. Zhao et al demonstrated that overexpressed SNHG5 significantly represses the progression of gastric cancer $(17,26)$. While, Damas et al uncovered 
A

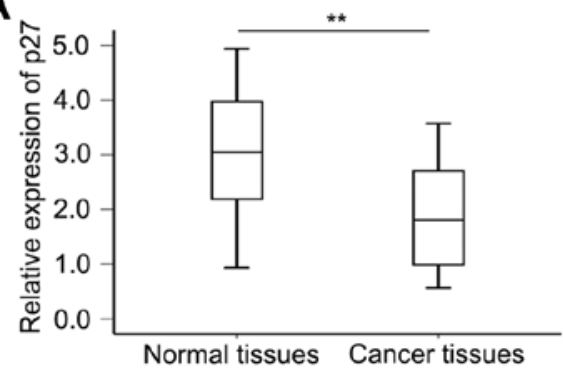

B

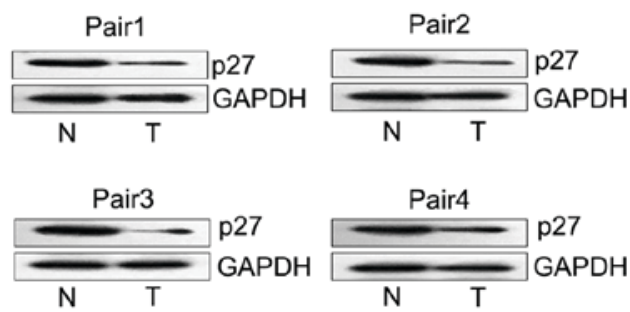

C

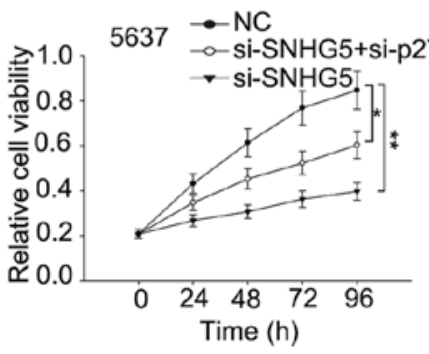

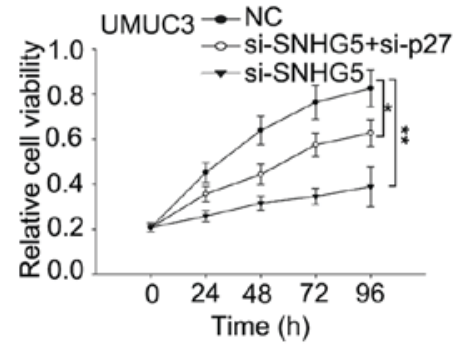

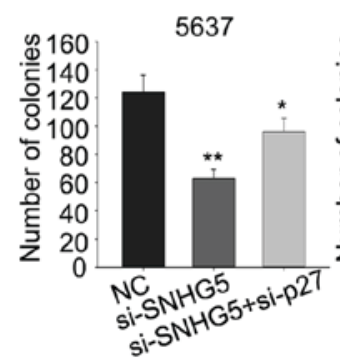

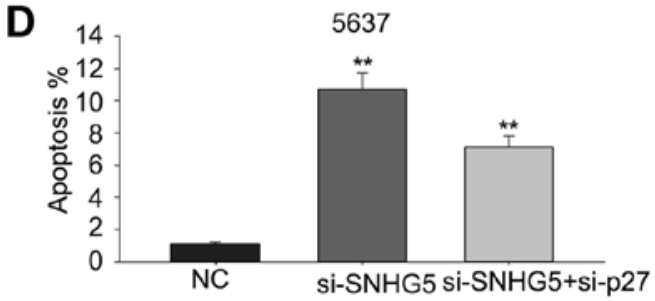

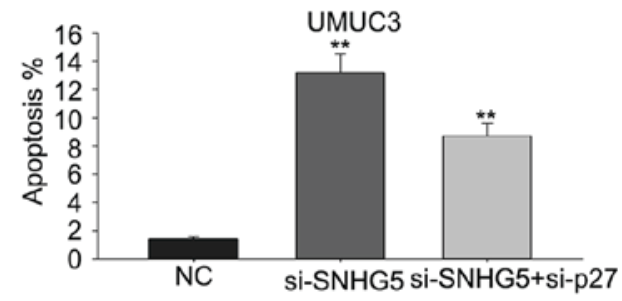

E
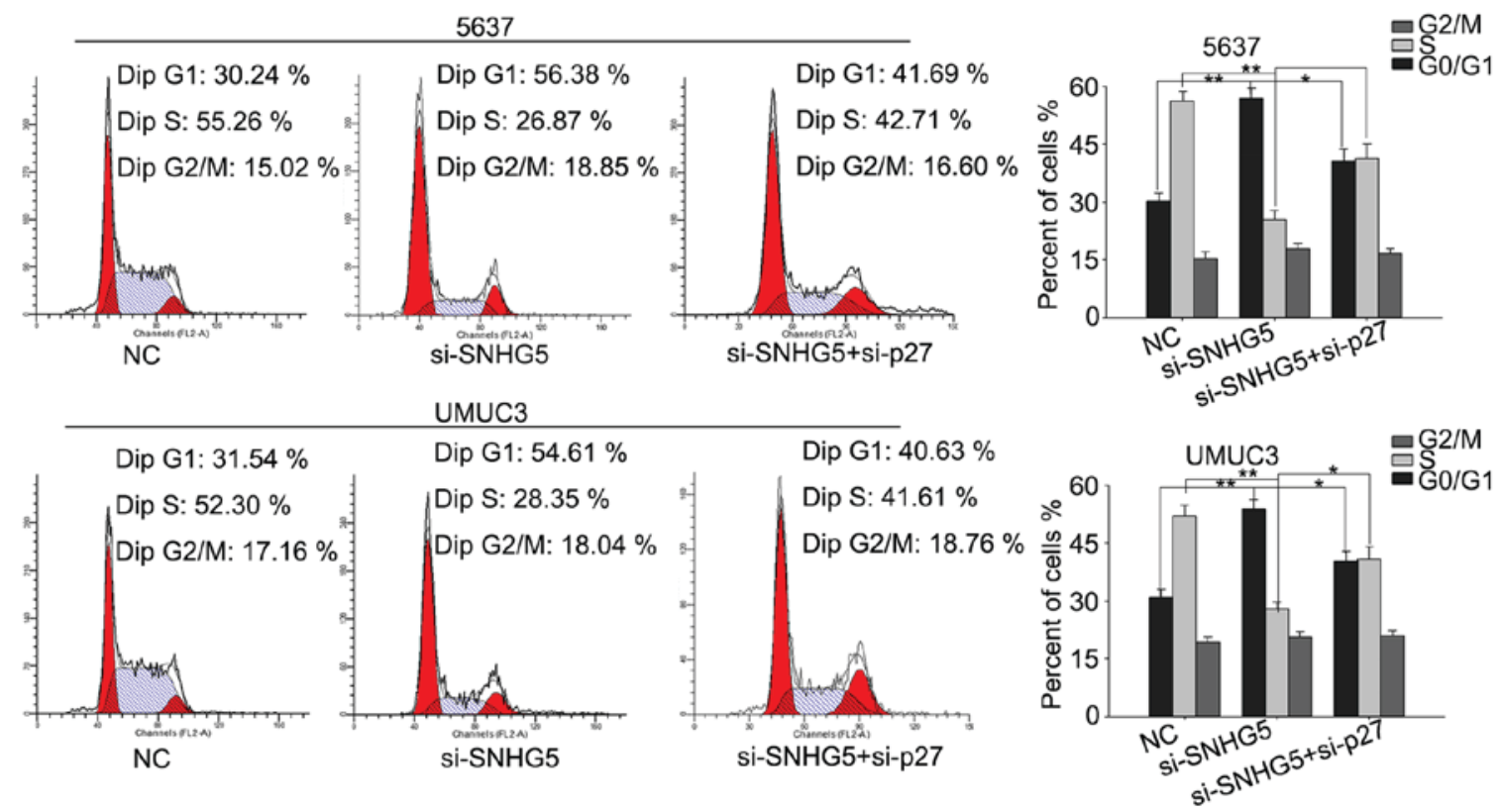

Figure 5. The oncogenic function of SNHG5 is in a p27-dependent manner. (A and B) Reverse transcription-quantitative polymerase chain reaction and western blot analysis were employed to determine the level of $p 27$ in the BC tissues and corresponding normal tissues. (C) MTT and colony-formation assays were performed to assess the proliferation ability of BC cells co-transfected with si-SNHG5 and si-p27. (D and E) Apoptosis rate and cell cycle distribution of cells co-transfected with si-SNHG5 and si-p27 were measured by flow cytometric analyses. Error bars represented the mean \pm standard deviation of at least three independent experiments. ${ }^{*} \mathrm{P}<0.05,{ }^{* *} \mathrm{P}<0.01$ vs. control group. SNHG5, small nucleolar RNA host gene 5; si small interfering; p27, cyclin-dependent kinase inhibitor 1B; BC, bladder carcinoma.

that high level of SNHG5 obviously promotes cell survival in colorectal cancer (16). Consistently, our study also presented tumor-promoting function of $S N H G 5$. As we known, the function of IncRNAs has the cancer-specificity, means that the role of same IncRNA was different in different cancer type. The different role of SNHG5 in different cancer-type profoundly explants tumor-type specificity of IncRNAs.

Collectively, our study presented that SNHG5 was significantly upregulated in $\mathrm{BC}$ tissues and cells lines. And molecular experiments revealed that SNHG5 exerted an 
oncogene functions in the genesis and progression of $\mathrm{BC}$, which provided a potential attractive therapeutic target for this malignancy.

\section{References}

1. Li N, Yang L, Wang H, Yi T, Jia X, Chen C and Xu P: miR-130a and miR-374a function as novel regulators of cisplatin resistance in human ovarian cancer A2780 cells. PLoS One 10: e0128886, 2015.

2. Yu H, Xue Y, Wang P, Liu X, Ma J, Zheng J, Li Z, Li Z, Cai H and Liu Y: Knockdown of long non-coding RNA XIST increases blood-tumor barrier permeability and inhibits glioma angiogenesis by targeting miR-137. Oncogenesis 6: e303, 2017.

3. Su J, Zhang E, Han L, Yin D, Liu Z, He X, Zhang Y, Lin F, Lin Q, Mao P, et al: Long noncoding RNA BLACAT1 indicates a poor prognosis of colorectal cancer and affects cell proliferation by epigenetically silencing of p15. Cell Death Dis 8: e2665, 2017.

4. Xu Y, Wang B, Zhang F, Wang A, Du X, Hu P, Zhu Y and Fang Z: Long non-coding RNA CCAT2 is associated with poor prognosis in hepatocellular carcinoma and promotes tumor metastasis by regulating Snail2-mediated epithelial-mesenchymal transition. Onco Targets Ther 10: 1191-1198, 2017.

5. Li W, Li H, Zhang L, Hu M, Li F, Deng J, An M, Wu S, Ma R, Lu J and Zhou Y: Long non-coding RNA LINC00672 contributes p53-mediated gene suppression and promotes endometrial cancer chemosensitivity. J Biol Chem 292: 5801-5813, 2017.

6. Wang JZ, Xu CL, Wu H and Shen SJ: IncRNA SNHG12 promotes cell growth and inhibits cell apoptosis in colorectal cancer cells. Braz J Med Biol Res 50: e6079, 2017.

7. Mang Y, Li L, Ran J,Zhang S, Liu J,Li L, Chen Y, Liu J, Gao Y and Ren G: Long noncoding RNA NEAT1 promotes cell proliferation and invasion by regulating hnRNP A2 expression in hepatocellular carcinoma cells. Onco Targets Ther 10: 1003-1016, 2017.

8. Li H, Jiang X and Niu X: Long non-coding RNA reprogramming (ROR) promotes cell proliferation in colorectal cancer via affecting P53. Med Sci Monit 23: 919-928, 2017.

9. Li X, Yang J, Sun L, Zhang L, Jiang Z, Puri P, Gurley EC, Lai G, Tang Y, Huang Z, et al: The role of long noncoding RNA H19 in gender disparity of cholestatic liver injury in multidrug resistance 2 gene knockout mice. Hepatology 66: 869-884, 2017.

10. Idogawa M, Ohashi T, Sasaki Y, Nakase H and Tokino T: Long non-coding RNA NEAT1 is a transcriptional target of p53 and modulates p53-induced transactivation and tumor-suppressor function 140: 2785-2791, 2017.

11. Zhou C, Huang C, Wang J, Huang H, Li J, Xie Q, Liu Y, Zhu J, Li Y, Zhang D, et al: LncRNA MEG3 downregulation mediated by DNMT3b contributes to nickel malignant transformation of human bronchial epithelial cells via modulating PHLPP1 transcription and HIF-1 $\alpha$ translation. Oncogene 36: 3878-3889, 2017.

12. Wang TH, Chan CW, Fang JY, Shih YM, Liu YW, Wang TV and Chen CY: 2-O-Methylmagnolol upregulates the long non-coding RNA, GAS5, and enhances apoptosis in skin cancer cells. Cell Death Dis 8: e2638, 2017.

13. Pei Z, Du X, Song Y, Fan L, Li F, Gao Y, Wu R, Chen Y, Li W, Zhou $\mathrm{H}$, et al: Down-regulation of $\ln \mathrm{RNA}$ CASC2 promotes cell proliferation and metastasis of bladder cancer by activation of the Wnt/ $\beta$-catenin signaling pathway. Oncotarget 8: 18145-18153, 2017.

14. Liu D, Li Y, Luo G, Xiao X, Tao D, Wu X, Wang M, Huang C, Wang L, Zeng F and Jiang G: LncRNA SPRY4-IT1 sponges miR-101-3p to promote proliferation and metastasis of bladder cancer cells through up-regulating EZH2. Cancer Lett 388: 281-291, 2017.
15. Pan J, Li X, Wu W, Xue M, Hou H, Zhai W and Chen W: Long non-coding RNA UCA1 promotes cisplatin/gemcitabine resistance through CREB modulating miR-196a-5p in bladder cancer cells. Cancer Lett 382: 64-76, 2016.

16. Damas ND, Marcatti M, Côme C, Christensen LL, Nielsen MM, Baumgartner R, Gylling HM, Maglieri G, Rundsten CF, Seemann SE, et al: SNHG5 promotes colorectal cancer cell survival by counteracting STAU1-mediated mRNA destabilization. Nat Commun 7: 13875, 2016.

17. Zhao L, Guo H, Zhou B, Feng J, Li Y, Han T, Liu L, Li L, Zhang S, Liu Y, et al: Long non-coding RNA SNHG5 suppresses gastric cancer progression by trapping MTA2 in the cytosol. Oncogene 35: 5770-5780, 2016

18. Zou G, Liu T, Guo L, Huang Y, Feng Y, Huang Q and Duan T: miR-145 modulates lncRNA-ROR and Sox 2 expression to maintain human amniotic epithelial stem cell pluripotency and $\beta$ islet-like cell differentiation efficiency. Gene 591: 48-57, 2016.

19. Zhu H, Lv Z, An C, Shi M, Pan W, Zhou L, Yang W and Yang M: Onco-lncRNA HOTAIR and its functional genetic variants in papillary thyroid carcinoma. Sci Rep 6: 31969, 2016.

20. Zheng H and Min J: Role of long noncoding RNA HOTAIR in the growth and apoptosis of osteosarcoma cell MG-63. Biomed Res Int 2016: 5757641, 2016.

21. Zhang XY, Zhang LX, Tian CJ, Tang XY, Zhao LM, Guo YL, Cheng DJ, Chen XL, Ma LJ and Chen ZC: LncRNAs BCYRN1 promoted the proliferation and migration of rat airway smooth muscle cells in asthma via upregulating the expression of transient receptor potential 1. Am J Transl Res 8: 3409-3418, 2016.

22. Zhang E, Han L, Yin D, He X, Hong L, Si X, Qiu M, Xu T, De W, Xu L, et al: H3K27 acetylation activated-long non-coding RNA CCAT1 affects cell proliferation and migration by regulating SPRY4 and HOXB13 expression in esophageal squamous cell carcinoma. Nucleic Acids Res 45: 3086-3101, 2017.

23. Xue M, Pang H, Li X, Li H, Pan J and Chen W: Long non-coding RNA urothelial cancer-associated 1 promotes bladder cancer cell migration and invasion by way of the hsa-miR-145-ZEB1/2-FSCN1 pathway. Cancer Sci 107: 18-27, 2016.

24. Zhang CZ: Long non-coding RNA FTH1P3 facilitates oral squamous cell carcinoma progression by acting as a molecular sponge of miR-224-5p to modulate fizzled 5 expression. Gene 607: 47-55, 2017.

25. Cui Y, Zhang F, Zhu C, Geng L, Tian T and Liu H: Upregulated lncRNA SNHG1 contributes to progression of non-small cell lung cancer through inhibition of miR-101-3p and activation of Wnt/ $\beta$-catenin signaling pathway. Oncotarget 8: 17785-17794, 2017.

26. Zhao L, Han T, Li Y, Sun J, Zhang S, Liu Y, Shan B, Zheng D and Shi J: The lncRNA SNHG5/miR-32 axis regulates gastric cancer cell proliferation and migration by targeting KLF4. Faseb J 31: 893-903, 2017. 